\title{
Definition of the Response to Initial Therapy with Radioiodine in Patients with Differentiated Thyroid Carcinoma: Basal or Stimulated Thyroglobulin?
}

Authors

Pedro Weslley Rosario, Gabriela Franco Mourão, Maria Regina Calsolari

Affiliation

Santa Casa de Belo Horizonte, Minas Gerais, Brazil

Key words

differentiated thyroid carcinoma, response to therapy, thyroglobulin, stimulation, recurrence

$\begin{array}{lr}\text { received } & 25.06 .2019 \\ \text { accepted } & 01.08 .2019\end{array}$

Bibliography

DOI https://doi.org/10.1055/a-0991-0231

Horm Metab Res 2019; 51: 634-638

(c) Georg Thieme Verlag KG Stuttgart - New York ISSN 0018-5043

Correspondence

Pedro Weslley Rosario, MD

Instituto de Ensino e Pesquisa da Santa Casa de Belo

Horizonte

Rua Domingos Vieira 590

Santa Efigênia

CEP 30150-240 Belo Horizonte

MG

Brazil

Tel.: + 55/313/2388 838, Fax: + 55/313/2130836

pedrowsrosario@gmail.com

\section{ABSTRACT}

Basal thyroglobulin (b-Tg) measured with second-generation assay or stimulated $\mathrm{Tg}(\mathrm{s}-\mathrm{Tg}$ ) can be used to define the response to therapy of differentiated thyroid carcinoma. However, they do not always define the same category and guidelines do not establish "if" or "when" s-Tg needs to be obtained. We studied 304 patients without clinically apparent disease or disease detected by neck ultrasonography and without anti-Tg antibodies 9-12 months after therapy. Based on b-Tg, 196 patients had an excellent response and $108 \mathrm{had}$ an indeterminate response. Based on $\mathrm{s}-\mathrm{Tg}$, a change in category occurred in $10.2 \%$ of the patients with an initial excellent response (all to indeterminate response) and in half the patients with an initial indeterminate response ( $44.4 \%$ to excellent response and $5.5 \%$ to biochemical incomplete response). One case of recurrence was observed among patients with an initial excellent response but whose response changed to indeterminate after $\mathrm{s}-\mathrm{Tg}$, while no disease was detected among those who remained in the initial category; however, this difference was not significant. In patients with an initial indeterminate response, no recurrence was detected among those whose response changed to excellent after $\mathrm{s}-\mathrm{Tg}$, while 11.1 and $33.3 \%$ of those who remained in the initial category or whose response changed to biochemical incomplete, respectively, had structural disease. This study suggest that, in low- or intermediate-risk patients, s-Tg better defines the response to therapy with ${ }^{131}$ I when it is classified as indeterminate based on b-Tg using second-generation assay. However, s-Tg is not necessary when $\mathrm{b}-\mathrm{Tg}$ defines the response as excellent.

\section{Introduction}

In patients with differentiated thyroid carcinoma (DTC) who received radioactive iodine (RAI) after total thyroidectomy, evaluation of the response to initial therapy starts with the measurement of basal thyroglobulin (b-Tg) and anti-Tg antibodies ( $\mathrm{TgAb}$ ) and neck ultrasonography (US) [1, 2]. In patients without an apparent tumor on US, especially low- or intermediate-risk cases, initial categorization of the response to therapy as excellent, indeterminate or incomplete is fundamental for management. Diagnostic wholebody scanning (DxWBS) is not necessary in the case of an excellent response [1 (Recommendation 66 - ATA Guidelines),2]. On the other hand, imaging methods other than US should be performed in patients with an initial biochemical incomplete response to rule out structural disease [2]. In the follow-up of low-risk patients with an excellent response, repetition of US is not necessary if b-Tg and TgAb remain negative [ 1 (Recommendation 65D - ATA Guidelines)]. The measurement of $\mathrm{b}-\mathrm{Tg}$ is recommended every $12-24$ months after an excellent response [ 1 (Recommendation $62 \mathrm{C}$ - ATA Guidelines),2] versus 6-12 months after an indeterminate or incomplete response [1 (Recommendation 62E - ATA Guidelines),2]. Repetition of stimulated $\mathrm{Tg}(\mathrm{s}-\mathrm{Tg})$ is not recommended after an excellent response [1 (Recommendation 63B - ATA Guidelines),2] but may be considered after indeterminate and biochemical incomplete responses accompanied by b-Tg reduction during follow-up [1 (Recommendation 63C - ATA Guidelines),2]. Finally, the serum TSH target $(<0.1$, between 0.1 and 0.5 , or between 0.5 and $2 \mathrm{mIU} / \mathrm{l}$ ) dif- 
fers among response categories [1 (Recommendation 70 - ATA Guidelines),2]. These differences exist because the risk of developing structural disease during follow-up is different for each of the three categories [1].

Basal Tg measured by a second-generation assay or s-Tg can be used to define the response to therapy [ 1 (Recommendation $63 \mathrm{~A}$ - ATA Guidelines),2]. However, s-Tg may not correspond to the same category as b-Tg, a fact that can lead to a change in category of the patient if the first is obtained. Unfortunately, current guidelines do not define "if" or "when" s-Tg should be measured when second-generation assays are used [1-4].

The aim of this study was to evaluate the change in the response to initial therapy category when s-Tg is obtained in order to define for which patients its measurement would be indicated, even when second-generation Tg assays are used. As this doubt especially applies to low- or intermediate-risk patients who received RAI and who do not have apparent disease on US or positive $\mathrm{TgAb}$, this was the population of the study.

\section{Compliance with ethical standards}

The study was approved by the Research Ethics Committee of our institution.

\section{Patients and Methods}

\section{Patients}

At our service, investigation after initial therapy of DTC starts with the measurement of b-Tg and $\mathrm{TgAb}$ and neck US. We do not obtain $\mathrm{s}$-Tg in patients not receiving RAI or patients treated with RAI when US already reveals a tumor or b-Tg is already elevated. Since $2006, \mathrm{Tg}$ has been measured with a second-generation assay [5]. Thus, the patients included in this study were those treated with total thyroidectomy and RAl who had b-Tg $\leq 1 \mathrm{ng} / \mathrm{ml}$ and US without abnormalities 9-12 months after initial therapy and s-Tg obtained on that occasion. Patients classified as high risk according to the American Thyroid Association (ATA) [1] or those with TgAb were excluded.

\section{Initial evaluation}

Based on b-Tg, the response to initial therapy was classified as excellent if $<0.2 \mathrm{ng} / \mathrm{ml}$ and as indeterminate if between 0.2 and $1 \mathrm{ng} / \mathrm{ml}$ [1-4]. Stimulated $\mathrm{Tg}$ was obtained $72 \mathrm{~h}$ after administration of the second rhTSH ampoule [6] or after 4 weeks without levothyroxine [with $\mathrm{TSH}>30 \mathrm{mIU} / \mathrm{l}$ ]. Based on $\mathrm{s}-\mathrm{Tg}$, the response to therapy was classified as excellent if $<1 \mathrm{ng} / \mathrm{ml}$, as indeterminate if between 1 and $10 \mathrm{ng} / \mathrm{ml}$, and as biochemical incomplete if $>10 \mathrm{ng} / \mathrm{ml}$ [1-4].

During initial evaluation, imaging methods other than US [neck, chest and mediastinal computed tomography (CT), fluorodeoxyglucose-positron emission tomography (FDG-PET)/CT] were performed if s-Tg $\geq 2 \mathrm{ng} / \mathrm{ml}$. Empirical ${ }^{131}$ I therapy was not performed in these patients.

\section{Follow-up}

The patients were maintained on 0.1-2 mIU/I TSH and were followed up by clinical examination, measurement of $\mathrm{Tg}$ and $\mathrm{TgAb}$ at intervals of 6-12 months, and annual neck US. Imaging methods other than US were performed if $\mathrm{b}-\mathrm{Tg} \geq 1 \mathrm{ng} / \mathrm{ml}$.

\section{Methods}

US was performed with a linear multifrequency transducer for morphological analysis (B-mode) and for power Doppler evaluation. All suspected lesions apparent on the scans $[7,8]$ were evaluated by US-guided fine-needle aspiration biopsy. CT with contrast was performed on 5-mm sequential sections. FDG-PET/CT was carried out according to a recommended protocol $[9,10]$. All images were analyzed by experienced Radiology or Nuclear Medicine specialists.

Structural disease was defined based on the imaging results, cytology or histology, and/or unequivocal ectopic uptake (excluding false-positive results) on post-therapy WBS (RxWBS) or FDG-PET/ CT. Tumor recurrence was defined as structural disease diagnosed after s-Tg measurement in patients without apparent disease in the initial evaluation.

Chemiluminescent assays were used for the measurement of $\mathrm{Tg}$ [Access Thyroglobulin Assay, Beckman Coulter, Fullerton, CA (functional sensitivity of $0.1 \mathrm{ng} / \mathrm{ml}$ at our laboratory [11]) and $\mathrm{TgAb}$ [Immulite 2000, Diagnostic Products Corporation, Los Angeles, CA (reference value of up to $40 \mathrm{IU} / \mathrm{ml}$ ) or ARCHITECT Anti-Tg, Abbott Laboratories, IL, USA (reference value of up to $4.11 \mathrm{IU} / \mathrm{ml}$ )].

\section{Statistical analysis}

Subgroups were defined by combining the category of response to therapy based on u-Tg with the category defined by s-Tg. Fisher's exact test or chi-squared test was used to detect differences in the rate of recurrence between the subgroups. These tests were also used to compare the rate of recurrence among low and intermediate risk patients. A p-value less than 0.05 was considered significant.

\section{Results}

\section{Patients}

We evaluated 304 patients with DTC who were initially treated with total thyroidectomy and RAI and who had no clinically apparent disease or disease detected on neck US and were negative for TgAb 9-12 months after initial therapy. Basal Tg ranged from undetectable to $1 \mathrm{ng} / \mathrm{ml}$ (median $0.11 \mathrm{ng} / \mathrm{ml}$ ). Based on b-Tg, 196 patients had an excellent response and 108 had an indeterminate response to therapy. The characteristics of the 304 patients are shown in - Table 1.

\section{Stimulated $\mathrm{Tg}$}

Based on s-Tg, a change in category occurred in $10.2 \%$ (20/196) of the patients with an excellent response (all to indeterminate response) and in half the patients with an indeterminate response (44.4\% to excellent response and $5.5 \%$ to biochemical incomplete response). The number of patients who changed the category of the response to therapy is shown in $>$ Fig. 1 .

\section{Frequency of recurrence}

After s-Tg measurement, the patients were followed up for 24-120 months (median 62 months).

Structural disease has so far been detected in 9 patients, including 8 with cervical metastases (detected by US in 6 and by FDG-PET in 2) and one with pulmonary metastases (detected by CT). Tumor 
- Table 1 Characteristics of the 304 patients.

\begin{tabular}{|l|l|}
\hline Sex & \\
\hline Female & $244(80.2 \%)$ \\
\hline Male & $60(19.7 \%)$ \\
\hline Age & $14-81$ years (mean 49 years) \\
\hline$\leq 55$ years & $198(65.1 \%)$ \\
\hline$>55$ years & $106(34.8 \%)$ \\
\hline Histology & $284(93.4 \%)$ \\
\hline Papillary & $12(4 \%)$ \\
\hline Follicular & $8(2.6 \%)$ \\
\hline Hurthle Cell & $91(30 \%)$ \\
\hline ATA initial risk & $213(70 \%)$ \\
\hline $\begin{array}{l}\text { Low risk } \\
\text { Intermediate risk }\end{array}$ & $22(7.2 \%) / 282(92.7 \%)$ \\
\hline $\begin{array}{l}\text { Initial therapy } \\
\text { Completion following lobectomy/prima- } \\
\text { ry total thyroidectomy }\end{array}$ & $123(40.4 \%) / 10(3.3 \%) / 171$ \\
\hline $\begin{array}{l}\text { Lymph node dissection: therapeutic/ } \\
\text { elective/not performed }\end{array}$ & $(56.2 \%)$ \\
\hline $\begin{array}{l}131 \\
\text { I therapy: } 30-50 \text { mCi/100 mCi-150mCi }\end{array}$ & $201(66.1 \%) / 103(33.9 \%)$ \\
\hline
\end{tabular}

ATA: American Thyroid Association. " All with tumors $>1 \mathrm{~cm}$ and none with the noninvasive encapsulated follicular variant.

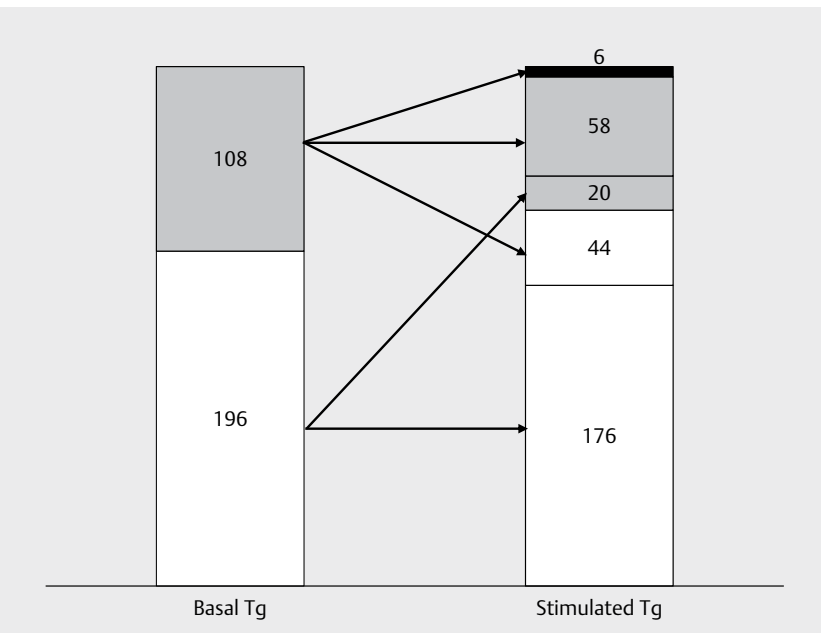

-Fig. 1 Patients who changed the category of the response to therapy. Excellent response (white boxes), Indeterminate response (gray boxes), Biochemical incomplete response (black box).

recurrence was detected for $6-72$ months (median 36 months) after s-Tg measurement. After surgery, six patients were free of disease (undetectable nonstimulated $\mathrm{Tg}$, negative $\mathrm{TgAb}$, US without anomalies) and one patient continued to have detectable nonstimulated $\mathrm{Tg}<1 \mathrm{ng} / \mathrm{ml}$ and no apparent tumor was detected by the imaging methods. In the last evaluation, two other patients had persistent disease but stable ( $\triangleright$ Table 2 ).
Using b-Tg for definition of the response to therapy, the recurrence rate was significantly lower for the excellent response category $(0.5 \%)$ compared to the indeterminate category $(7.4 \%)$ $(p=0.001)$. Using s-Tg, this difference was also observed [0 vs. $9.4 \%$ $(p=0.0001)]$. No difference was found in the frequency of recurrence between the excellent $[0.5$ vs. $0 \%(p=0.46)]$ or indeterminate responses $[7.4$ vs. $9.4 \%(p=0.78)]$ using either $b-T g$ or $s-T g$, respectively.

One case of recurrence was observed among patients with an initial excellent response (based on b-Tg) but whose response changed to indeterminate after $\mathrm{s}-\mathrm{Tg}$, while no disease was detected in those who remained in the initial category; however, this difference was not significant $(p=0.1)$.

In patients with an indeterminate response (based on b-Tg), no case of recurrence was detected among those whose response changed to excellent after s-Tg, while 11.1 and $33.3 \%$ of those who remained in the initial category or whose response changed to biochemical incomplete, respectively, had structural disease. The frequency was significantly lower in the first group.

- Table 1 shows the rate of recurrence in each subgroup. The frequency of structural disease, percentage of change in category or recurrence rates did not differ between low- and intermediate risk patients and these patients were therefore grouped.

\section{Discussion}

We first address some characteristics that differentiate this work from previous studies that also reported the results of s-Tg in patients with low b-Tg measured with second-generation assays. To increase clinical applicability, the present study evaluated the cut offs currently proposed by ATA [1] and other societies [2, 4, 12, 13] to define the response to thyroidectomy followed by RAl, that is, 0.2 and $1 \mathrm{ng} / \mathrm{ml}$ for b-Tg and 1 and $10 \mathrm{ng} / \mathrm{ml}$ for s-Tg. As also currently recommended, US was obtained in all patients during initial evaluation $[1-4,12,13]$. This imaging method is the minimum required to rule out structural disease $[1-4,12,13]$. In addition, the omission of US during initial evaluation may overestimate the longterm frequency of neck recurrence. Empirical ${ }^{131}$ I therapy was not performed in our patients. We evaluated not only the results of s-Tg but also the recurrence rate in different subgroups after follow-up for up to 120 months. Finally, high-risk patients were excluded since even an excellent response in this group might be associated with a considerable frequency of persistent or recurrent disease [1-3, 14-16].

In the group with an initial excellent response to therapy based on b-Tg, all 20 patients who changed category after s-Tg were classified as indeterminate response. When this change occurred, the recurrence rate was $5 \%$. Brassard et al. [17] found recurrence in $11 \%$ of 26 patients with $\mathrm{b}-\mathrm{Tg} \leq 0.27 \mathrm{ng} / \mathrm{ml}$ but $\mathrm{s}-\mathrm{Tg}>1.4 \mathrm{ng} / \mathrm{ml}$. The authors of that study did not routinely perform US to rule out apparent disease already during initial evaluation, as recommended $[1-4,12,13]$. A rate of structural recurrence of about $10 \%$ has also been reported in three other studies involving a smaller number of patients initially without US abnormalities and with $\mathrm{b}-\mathrm{Tg} \leq 0.1 \mathrm{ng} /$ $\mathrm{ml}$ [18], $0.15 \mathrm{ng} / \mathrm{ml}$ [19], or $0.2 \mathrm{ng} / \mathrm{ml}$ [20], but s-Tg $>1 \mathrm{ng} / \mathrm{ml}$. In contrast, Hu et al. [21] detected no recurrence after 36 months in 25 patients with $\mathrm{b}-\mathrm{Tg} \leq 0.2 \mathrm{ng} / \mathrm{ml}$ but $\mathrm{s}-\mathrm{Tg}>1 \mathrm{ng} / \mathrm{ml}$. Although the 
- Table 2 Frequency of tumor recurrence considering the response to therapy based on b-Tg and s-Tg.

Response based on b-Tg [recurrence (\%)]

Excellent [1/196 (0.5\%)]

Indeterminate $[8 / 108(7.4 \%)]$

Total $[9 / 304(3 \%)]$
Response based on s-Tg [recurrence (\%)]

\begin{tabular}{|l|l|l|}
\hline Excellent & Indeterminate & Incomplete \\
\hline $0 / 176$ & $1 / 20(5 \%)$ & \\
\hline $0 / 48$ & $6 / 54(11.1 \%)$ & $2 / 6(33.3 \%)$ \\
\hline $0 / 224$ & $7 / 74(9.4 \%)$ & $2 / 6(33.3 \%)$ \\
\hline
\end{tabular}

b-Tg: Basal thyroglobulin; s-Tg: Stimulated thyroglobulin.

change from an excellent to indeterminate response may identify a subgroup of patients with a higher recurrence risk than those whose $\mathrm{s}$-Tg confirms an excellent response, this conversion occurs in a minority of patients (present study, [17-22]). This low frequency of change in category, together with the results above showing that, even if conversion occurs, only $10 \%$ of patients or less develop recurrence, renders the measurement of s-Tg unattractive in patients with an excellent response to initial therapy based on b-Tg. In addition, one must consider the cost of rhTSH or the risks and complications of acute hypothyroidism [23].

Among the 108 patients with an indeterminate response based on b-Tg, a change to the excellent response category after s-Tg was observed in 44\%. In agreement with this finding, Brassard et al. [17] showed that $41 \%$ of 117 patients with $\mathrm{b}-\mathrm{Tg}>0.27 \mathrm{ng} / \mathrm{ml}$ had $\mathrm{s}-\mathrm{Tg}<1.4 \mathrm{ng} / \mathrm{ml}$, Malandrino et al. [19] that $36 \%$ of 69 patients with b-Tg between 0.15 and $1 \mathrm{ng} / \mathrm{ml}$ had s-Tg $\leq 1 \mathrm{ng} / \mathrm{ml}$, and Trimboli et al. [24] that $39 \%$ of 57 patients with $b-T g>0.1 \mathrm{ng} / \mathrm{ml}$ had $\mathrm{s}-\mathrm{Tg}$ $\leq 1 \mathrm{ng} / \mathrm{ml}$. Even more important, the risk of recurrence in patients whose response changed from indeterminate to excellent after s-Tg was compatible with the last category. None of the 48 patients developed recurrence, similar to the observation in patients in which both, b-Tg and s-Tg, indicated an excellent response. Despite the use of a cut off that was slightly different from the currently recommended $[1-4,12,13]$, similar results have been reported by Brassard et al. [17] who demonstrated recurrence in only $1 / 48$ patients ( $2 \%$ ) with b-Tg $>0.27 \mathrm{ng} / \mathrm{ml}$ but s- $\mathrm{Tg}<1.4 \mathrm{ng} / \mathrm{ml}$; by Malandrino et al. [19] who found no short-term recurrence in 25 patients with b-Tg $>0.15 \mathrm{ng} / \mathrm{ml}$ but initial US showing no tumor and $\mathrm{s}-\mathrm{Tg} \leq 1 \mathrm{ng} /$ $\mathrm{ml}$; and by Trimboli et al. [24] who did not detect disease in 22 patients with $\mathrm{b}-\mathrm{Tg}>0.1 \mathrm{ng} / \mathrm{ml}$ but $\mathrm{s}-\mathrm{Tg} \leq 1 \mathrm{ng} / \mathrm{ml}$. Different from these results, the frequency of recurrence was $7.5 \%$ in patients with an indeterminate response based only on b-Tg and $11 \%$ when both, b-Tg and s-Tg, defined this category. In agreement, a recent study also showed biochemical or structural recurrence after follow-up for 68 months (median) in $9 \%$ of patients initially classified as having an indeterminate response based on both b-Tg and s-Tg [25].

Considering the frequency of change to an excellent response and the consequences of this change, obtaining s-Tg seems to be interesting in patients with an initial indeterminate response based on b-Tg. With this change in category, these patients may no longer require to be submitted to US repetition if $\mathrm{b}-\mathrm{Tg}$ and $\mathrm{TgAb}$ remain stable [1], the interval of Tg measurement can be increased to every 12-24 months [ 1,2$]$, repetition of s-Tg is not recommended [1, 2], and TSH suppression is not necessary [1,2]. Secondly, in some of our patients, the indeterminate response changed to biochemical incomplete after s-Tg, a finding that requires more frequent follow-up, better investigation, and more intense TSH suppression [1-3]. Indeed, although the number of patients with this change in category after s-Tg was small, the risk of recurrence was high in this group.

We recognize that the retrospective design of the study is a limitation; however, all patients were seen at the same institution, were evaluated by the same professionals, and were submitted to the same follow-up and investigation protocol. Notably, patients with elevated $\mathrm{Tg}$ (initial s-Tg $\geq 2 \mathrm{ng} / \mathrm{ml}$ or $\mathrm{b}-\mathrm{Tg} \geq 1 \mathrm{ng} / \mathrm{ml}$ during follow-up) were investigated by neck US, CT, FDG-PET/CT [26, 27], a highly sensitive combination for the detection of metastases [27]. Another limitation is the time of follow-up, with a median of 60 months; however, it is known that 3/4 of recurrences occur in these first years [17]. In addition, the time was similar among the groups whose recurrence rates were compared (data not shown). Although we included low-risk patients, all they had tumors $>1 \mathrm{~cm}$, none with the noninvasive encapsulated follicular variant, and the frequency of structural disease, percentage of change in category or recurrence rates did not differ between low- and intermediate risk patients. Finally, one group not evaluated in the present study were patients with a biochemical incomplete response based on b-Tg.

The results of this study suggest that in low- or intermediate-risk patients treated with RAI, s-Tg better defines the response to therapy when it is classified as indeterminate based on b-Tg using a second-generation assay. However, s-Tg is not necessary when b-Tg defines the response to therapy as excellent.

\section{Funding}

This work was supported by the National Council for Scientific and Technological Development (CNPq).

\section{Conflict of Interest}

The authors declare that they have no conflict of interest. 


\section{References}

[1] Haugen BR, Alexander EK, Bible KC et al. 2015 American Thyroid Association Management Guidelines for Adult Patients with Thyroid Nodules and Differentiated Thyroid Cancer: The American Thyroid Association Guidelines Task Force on Thyroid Nodules and Differentiated Thyroid Cancer. Thyroid 2016; 26: 1-133

[2] Pacini F, Basolo F, Bellantone R et al. Italian consensus on diagnosis and treatment of differentiated thyroid cancer: Joint statements of six Italian societies. J Endocrinol Invest 2018; 41: 849-876

[3] Momesso DP, Tuttle RM. Update on differentiated thyroid cancer staging. Endocrinol Metab Clin North Am 2014; 43: 401-421

[4] Zerdoud S, Giraudet AL, Leboulleux S et al. Radioactive iodine therapy, molecular imaging and serum biomarkers for differentiated thyroid cancer: 2017 Guidelines of the French Societies of nuclear medicine, endocrinology, pathology, biology, endocrine surgery and head and neck surgery. Ann Endocrinol (Paris) 2017; 78: 162-175

[5] Rosario PW, Purisch S. Does a highly sensitive thyroglobulin (Tg) assay change the clinical management of low-risk patients with thyroid cancer with $\mathrm{Tg}$ on $\mathrm{T} 4<1 \mathrm{ng} / \mathrm{ml}$ determined by traditional assays? Clin Endocrinol (Oxf) 2008; 68: 338-342

[6] Rosario PW, Siman TL, Calsolari MR. Day 3 thyroglobulin $\leq 1 \mathrm{ng} / \mathrm{ml}$ after recombinant human TSH just prior to radioactive iodine is predictive of low risk for persistent/recurrent disease in patients with papillary thyroid carcinoma. Endocrine 2015; 49: 170-174

[7] Rosario PW, de Faria S, Bicalho L et al. Ultrasonographic differentiation between metastatic and benign lymph nodes in patients with papillary thyroid carcinoma. J Ultrasound Med 2005; 24: 1385-1389

[8] Rosario PW, Tavares WC, Borges MA et al. Ultrasonographic differentiation of cervical lymph nodes in patients with papillary thyroid carcinoma after thyroidectomy and radioiodine ablation: A prospective study. Endocr Pract 2014; 20: 293-298

[9] Delbeke D, Coleman RE, Guiberteau M] et al. Procedure Guideline for SPECT/CT Imaging 1.0. J Nucl Med 2006; 47: 1227-1234

[10] Boellaard R, O'Doherty MJ, Weber WA et al. FDG PET and PET/CT: EANM procedure guidelines for tumour PET imaging: version 1.0. Eur J Nucl Med Mol Imaging 2010: 37: 181-200

[11] Rosario PW, Mourão GF, Siman TL et al. Serum thyroglobulin measured with a second-generation assay in patients undergoing total thyroidectomy without radioiodine remnant ablation: A prospective study. Thyroid 2015; 25: 769-775

[12] Beltsevich DG, Vanushko VE, Rumiantsev PO et al. 2017 Russian clinical practice guidelines for differentiated thyroid cancer diagnosis and treatment. Endocr Surg 2017; 11: 6-27

[13] Jarząb B, Dedecjus M, Słowińska-Klencka D et al. Guidelines of Polish National Societies Diagnostics and Treatment of Thyroid Carcinoma. 2018 Update Endokrynol Pol 2018; 69: 34-74

[14] Tuttle RM, Tala H, Shah J et al. Estimating risk of recurrence in differentiated thyroid cancer after total thyroidectomy and radioactive iodine remnant ablation: Using response to therapy variables to modify the initial risk estimates predicted by the New American Thyroid Association staging system. Thyroid 2010; 20: 1341-1349
[15] Kim TH, Ki CS, Kim HS et al. Refining dynamic risk stratification and prognostic groups for differentiated thyroid cancer with tert promoter mutations. J Clin Endocrinol Metab 2017; 102: 1757-1764

[16] van Velsen EFS, Stegenga M, van Kemenade FJ et al. Evaluating the 2015 American Thyroid Association risk stratification system in high risk papillary and follicular thyroid cancer patients. Thyroid 2019, doi:10.1089/thy.2019.0053

[17] Brassard M, Borget I, Edet-Sanson A et al. Long-term follow-up of patients with papillary and follicular thyroid cancer: A prospective study on 715 patients. J Clin Endocrinol Metab 2011; 96: 1352-1359

[18] Chindris AM, Diehl NN, Crook JE et al. Undetectable sensitive serum thyroglobulin $(<0.1 \mathrm{ng} / \mathrm{ml})$ in 163 patients with follicular cell-derived thyroid cancer: Results of rhTSH stimulation and neck ultrasonography and long-term biochemical and clinical follow-up. J Clin Endocrinol Metab 2012; 97: 2714-2723

[19] Malandrino P, Latina A, Marescalco S et al. Risk-adapted management of differentiated thyroid cancer assessed by a sensitive measurement of basal serum thyroglobulin. J Clin Endocrinol Metab 2011; 96: 1703-1709

[20] Castagna MG, Tala Jury HP, Cipri C et al. The use of ultrasensitive thyroglobulin assays reduces but does not abolish the need for TSH stimulation in patients with differentiated thyroid carcinoma. J Endocrinol Invest 2011; 34: e219-223

[21] Hu HY, Liang J, Zhang T et al. Suppressed thyroglobulin performs better than stimulated thyroglobulin in defining an excellent response in patients with differentiated thyroid cancer. Nucl Med Commun 2018; 39: 247-251

[22] Spencer C, Fatemi S, Singer P et al. Serum basal thyroglobulin measured by a second-generation assay correlates with the recombinant human thyrotropin-stimulated thyroglobulin response in patients treated for differentiated thyroid cancer. Thyroid 2010; 20: 587-595

[23] Rosário PW, Fagundes TA, Rezende LL et al. Assessing hypothyroidism in the preparation of patients with thyroid cancer: Cardiovascular risk, renal function, drug metabolism, persistence of elevated thyroid-stimulating hormone, and absence from work. Endocrinologist 2006; 16: 25-29

[24] Trimboli P, Imperiali M, Piccardo A et al. Multicentre clinical evaluation of the new highly sensitive Elecsys ${ }^{\circledR}$ thyroglobulin II assay in patients with differentiated thyroid carcinoma. Clin Endocrinol (Oxf) 2018; 88: 295-302

[25] Malandrino P, Tumino D, Russo $M$ et al. Surveillance of patients with differentiated thyroid cancer and indeterminate response: $\mathrm{A}$ longitudinal study on basal thyroglobulin trend. J Endocrinol Invest 2019, doi:10.1007/s40618-019-01044-3

[26] Rosario PW, Furtado M, de S, Mineiro Filho AF et al. Value of diagnostic radioiodine whole-body scanning after initial therapy in patients with differentiated thyroid cancer at intermediate and high risk for recurrence. Thyroid 2012; 22: 1165-1169

[27] Rosario PW, Mourão GF, dos Santos JB et al. Is empirical radioactive iodine therapy still a valid approach to patients with thyroid cancer and elevated thyroglobulin? Thyroid 2014; 24: 533-536 